\title{
Integration of transcriptomics data into a genome-scale metabolic model of the methanotrophic bacterium Methylotuvimicrobium alcaliphilum $20 \mathrm{Z}^{\mathrm{R}}$
}

\author{
Mikhail A. Kulyashov \\ BIOSOFT.RU, LLC \\ Novosibirsk State University \\ Institute of Cytology and Genetics \\ SB RAS, Institute of Computational \\ Technologies SB RAS \\ Novosibirsk, Russia \\ m.kulyashov@mail.ru \\ Semyon K. Kolmykov \\ BIOSOFT.RU, LLC \\ Institute of Computational Technologies \\ SB RAS, Institute of Cytology and \\ Genetics SB RAS, Novosibirsk, Russia \\ semyonk@developmentontheedge.com
}

\author{
Ivan S. Evshin \\ BIOSOFT.RU, LLC \\ Institute of Computational Technologies \\ SB RAS, \\ Novosibirsk, Russia \\ ivan@developmentontheedge.com \\ Tamara M. Khlebodarova \\ Kurchatov Genomics Center, \\ Institute of Cytology and Genetics \\ SB RAS, \\ Novosibirsk, Russia \\ tamara@bionet.nsc.ru
}

\author{
Nikita V. Ivanisenko \\ Kurchatov Genomics Center, \\ Institute of Cytology and Genetics \\ SB RAS, Novosibirsk, Russia \\ n.ivanisenko@gmail.com \\ Ilya R. Akberdin \\ BIOSOFT.RU, LLC \\ Institute of Cytology and Genetics \\ SB RAS, \\ Novosibirsk, Russia \\ Novosibirsk State University \\ Novosibirsk, Russia \\ akberdinir@gmail.com
}

\begin{abstract}
Aerobic methane-oxidizing bacteria or methanotrophs have the unique ability to grow on methane as their sole source of carbon and energy. The main metabolic steps of the methane utilization by microorganisms have been identified and well-studied to date. However, a detailed understanding of molecular genetic mechanisms that provide an adaptive response at the level of transcription regulation to various growth conditions, high and low $\mathrm{pH}$, temperature, and salinity is still elusive. To solve the issue we have conducted a detailed theoretical study of the molecular mechanisms of gene expression regulation in the bacterium Methylotuvimicrobium alcaliphilum $20 \mathrm{Z}^{\mathrm{R}}$ (hereinafter $20 \mathrm{ZR}$ ) based on the integration of original omics data into genome-scale metabolic model of the 20ZR.
\end{abstract}

Keywords - methane, methanotrophs, central metabolism, transcription, regulation, genome-scale modeling, Methylotuvimicrobium alcaliphilum $20 Z^{R}$

\section{Introduction}

Methane is a valuable source of energy, but on the other hand, it is a significant global product of recyclable waste and one of the most dangerous greenhouse gas [1]. In turn, methane is a promising carbon source for biosynthesis of biotechnologically useful compounds using aerobic methanotrophic bacteria as biocatalysts, and its oxidation by microbial communities of methanotrophic organisms is, in fact, the only biological mechanism for regulation and reduction of its content in the atmosphere [2-4].

In last decades, laboratory isolates of new pure cultures of methanotrophs were discovered and obtained, including facultative methanotrophs, extremophilic species and anaerobes, which greatly expanded our ideas and knowledge about both the taxonomic diversity of these microorganisms and their physiological capabilities [5-7]. Among them, haloalkaliphilic aerobic methanotrophs like 20ZR are standing out as the most promising microbial "factories" in industrial research as new sources of enzymes and protein based materials that are resistant to high salt and $\mathrm{pH}$ levels, and natural producers of amino acids, sugars, and osmoprotectants [8-11].
Over the past two decades of intensive experimentaltheoretical studies in the field of methanotrophy outstanding progress has been made in understanding of mechanisms for methane capture from the environment by methaneconsuming bacteria, identification of key metabolic steps of its utilization and the impact of various factors on both methane consumption rate and activity of metabolic pathways for its bioconversion. However, detailed understanding of molecular regulatory mechanisms and diversity of regulatory relationships in molecular-genetic machinery of methanotrophs (metabolic systems transporters and donors of electrons for the first methane oxidation reaction; influence of the specific intracytoplasmic membrane structure on rate and mechanisms of enzymatic reactions; mechanisms and ability for methanotrophic fermentation; the impact of quorum sensing on metabolic modes) has still been elusive. Elucidation of these fundamental principles of the methane utilization and bioconversion will provide economically reasonable use of methanotrophs in industrial biotechnology [4; 12].

Recent advances in high throughput experimental technologies and its application to investigations of structural-functional organization of methanotrophs metabolism not only resulted in significant progress in understanding of crucial mechanisms of the methane utilization by these bacteria, but also led to the establishment of a holistic vision of all cellular functions of methanotrophs on different hierarchical level of their organization (genome, transcriptome, proteome and metabolome levels). The latter, to a large extent, is due to the possibility to use modern methods of bioinformatics and systems biology, which allow ones to integrate accumulated datasets and bulk of knowledge on molecular-genetic systems of the methane bioconversion into mathematical models [13-14]. Developed genome-scale metabolic models for methane-consuming bacteria, in turn, enable both in silico predictions of features of metabolic systems of the methane utilization for a certain representative of the aerobic methanotrophs and evaluation of their biotechnological capabilities as a producers of valueadded target compounds [15-24]. 
Methods

Transcriptomics data analysis

To analyze original transcriptomic data on the growth of M. alcaliphilum $20 Z^{R}$ under various cultivation conditions, obtained as part of a collaboration with Prof. Marina Kalyuzhnaya (San Diego California State University, USA), the already published protocol [25] has been used.

Development of the genome-scale metabolic model

Original transcriptomic data [25] have be incorporated into our previously developed metabolic flux model of Methylotuvimicrobium alcaliphilum 20Z ${ }^{R}$ [20] using the COBRAme approach [26], which is a part of the popular tool for the development and analysis of genome-scale metabolic models, COBRApy [27].

\section{Results}

Original published transcriptomics data generated in different growth conditions of the methanotroph's cultivation have been integrated into earlier developed genome-scale $20 Z^{R}$ metabolic model [20]. It is the first attempt to integrate this type of experimental data into the metabolic model of methanotrophic bacterium. Extended version of the model have enabled more precise predictions for growth of the methanotroph, its metabolic potential as a producer of target biotech compounds depending on both growth conditions (copper and/or lanthanum presence, methane or methanol as a carbon source) and corresponding levels of genes expression.

\section{REFERENCES}

[1] S.E.M. Fletcher and H. Schaefer, "Rising methane: A new climate challenge,” Science, vol. 364, № 6444, pp. 932-933, 2019.

[2] P.J. Strong, M. Kalyuzhnaya, J. Silverman and W.P. Clarke, “A methanotroph-based biorefinery: potential scenarios for generating multiple products from a single fermentation," Bioresource Technology, vol. 215, pp. 314-323, 2016.

[3] S. Cantera, S. Bordel, R. Lebrero, J. Gancedo, P.A. García-Encina, and R. Muñoz, "Bio-conversion of methane into high profit margin compounds: an innovative, environmentally friendly and costeffective platform for methane abatement," World Journal of Microbiology and Biotechnology, vol. 35, № 1, p.16, 2019.

[4] M.G. Kalyuzhnaya, D. Collins and L. Chistoserdova, "Microbial Cycling of Methane," 2019.

[5] Y.A. Trotsenko and J.C. Murrell, "Metabolic aspects of aerobic obligate methanotrophy," Advances in applied microbiology, vol. 63, pp.183-229, 2008.

[6] S.N. Dedysh and C. Knief, "Diversity and phylogeny of described aerobic methanotrophs," in Methane Biocatalysis: Paving the Way to Sustainability, Springer, Cham, 2018, pp. 17-42.

[7] M.G. Kalyuzhnaya, O.A. Gomez and J.C. Murrell, "The methaneoxidizing bacteria (methanotrophs)," in Taxonomy, Genomics and Ecophysiology of Hydrocarbon-Degrading Microbes, 2019, pp.1-34.

[8] V.N. Khmelenina, M.G. Kalyuzhnaya, N.G. Starostina, N.E. Suzina, and Y.A. Trotsenko, "Isolation and characterization of halotolerant alkaliphilic methanotrophic bacteria from Tuva soda lakes," Current Microbiology, vol. 35, № 5, pp.257-261, 1997.

[9] Y.A. Trotsenko and V.N. Khmelenina, "The biology and osmoadaptation of haloalkaliphilic methanotrophs," Microbiology, vol. 71, № 2, pp. 123-132, 2002.

[10] C.A. Henard and M.T. Guarnieri, "Metabolic Engineering of Methanotrophic Bacteria for Industrial Biomanufacturing," in Methane Biocatalysis: Paving the Way to Sustainability, Springer, Cham., 2018, pp. 117-132.
[11] S. Nariya and M.G. Kalyuzhnaya, "Diversity, Physiology, and Biotechnological Potential of Halo (alkali) philic MethaneConsuming Bacteria," in Methanotrophs, Springer, Cham., 2019, pp. 139-161.

[12] L. Chistoserdova, "Methanotrophy: An Evolving Field," in Methane Biocatalysis: Paving the Way to Sustainability, Springer, Cham., 2018, pp. 1-15.

[13] I.R. Akberdin, M. Thompson and M.G. Kalyuzhnaya, "Systems Biology and Metabolic Modeling of C 1-Metabolism," in Methane biocatalysis: Paving the way to sustainability, Springer, Cham., 2018, pp. 99-115.

[14] Y. Zheng and L. Chistoserdova, "Multi-omics Understanding of Methanotrophs," in Methanotrophs, Springer, Cham., 2019. pp. 121138.

[15] M.G. Kalyuzhnaya, S. Yang, O.N. Rozova, N.E. Smalley, J. Clubb, A. Lamb, G.N. Gowda, D. Raftery, Y. Fu, F. Bringel and S. Vuilleumier, "Highly efficient methane biocatalysis revealed in a methanotrophic bacterium," Nature communications, vol. 4, p.2785, 2013.

[16] A. de la Torre, A. Metivier, F. Chu, L.M. Laurens, D.A. Beck, P.T. Pienkos, M.E. Lidstrom and M.G. Kalyuzhnaya, "Genome-scale metabolic reconstructions and theoretical investigation of methane conversion in Methylomicrobium buryatense strain 5G (B1)," Microbial cell factories, vol. 14, № 1, p. 188, 2015.

[17] M.G. Kalyuzhnaya, A.W. Puri and M.E. Lidstrom, "Metabolic engineering in methanotrophic bacteria," Metabolic engineering, vol. 29, pp. 142-152, 2015.

[18] A. Demidenko, I.R. Akberdin, M. Allemann, E.E. Allen and M.G. Kalyuzhnaya, "Fatty acid biosynthesis pathways in Methylomicrobium buryatense 5G (B1)," Frontiers in microbiology, vol. 7, p. 2167, 2017.

[19] A. Gilman, Y. Fu, M. Hendershott, F. Chu, A.W. Puri, A.L. Smith, M. Pesesky, R. Lieberman, D.A. Beck and M.E. Lidstrom, "Oxygenlimited metabolism in the methanotroph Methylomicrobium buryatense 5GB1C," PeerJ, vol. 5, p. e3945, 2017.

[20] I.R. Akberdin, M. Thompson, R. Hamilton, N. Desai, D. Alexander, C.A. Henard, M.T. Guarnieri and M.G. Kalyuzhnaya, "Methane utilization in Methylomicrobium alcaliphilum 202R: a systems approach," Scientific reports, vol. 8, № 1, p. 2512, 2018.

[21] C. Lieven, L.A. Petersen, S.B. Jørgensen, K.V. Gernaey, M.J. Herrgard and N. Sonnenschein, "A genome-scale metabolic model for Methylococcus capsulatus (Bath) suggests reduced efficiency electron transfer to the particulate methane monooxygenase," Frontiers in microbiology, vol. 9, p.2947, 2018.

[22] A.D. Nguyen, J.Y. Park, I.Y. Hwang, R. Hamilton, M.G. Kalyuzhnaya, D. Kim and E.Y. Lee, "Genome-scale evaluation of core one-carbon metabolism in gammaproteobacterial methanotrophs grown on methane and methanol,". Metabolic Engineering, vol. 57, pp. 1-12, 2019.

[23] S. Bordel, , Y. Rodríguez, A. Hakobyan, E. Rodríguez, R. Lebrero and R. Muñoz, "Genome scale metabolic modeling reveals the metabolic potential of three Type II methanotrophs of the genus Methylocystis," Metabolic engineering, vol. 54, pp. 191-199, 2019.

[24] C.A. Henard, I.R. Akberdin, M.G. Kalyuzhnaya and M.T. Guarnieri, "Muconic Acid Production from Methane using Rationallyengineered Methanotrophic Biocatalysts," Green Chemistry, vol. 21, № 24, pp.6731-6737, 2019.

[25] I. Akberdin, D. Collins, R. Hamilton, D.Y. Oshchepkov, A. Shukla, C. Nicora, E. Nakayaku, J.N. Adkins and M.G. Kalyuzhanaya, "Rare Earth Elements Alter Redox Balance in Methylomicrobium alcaliphilum 20ZR ," Frontiers in microbiology, vol. 9, p. 2735, 2018.

[26] C.J. Lloyd, A. Ebrahim, L. Yang, Z.A. King, E. Catoiu, E.J. O’Brien, J.K. Liu and B.O. Palsson, "COBRAme: A computational framework for genome-scale models of metabolism and gene expression," PLoS computational biology, vol. 14, № 7, p. e1006302, 2018.

[27] A. Ebrahim, J.A. Lerman, B.O. Palsson and D.R. Hyduke, "COBRApy: constraints-based reconstruction and analysis for python,” BMC systems biology, vol. 7, № 1, p. 74, 2013. 